\title{
The Cyclopropylcarbinyl Rearrangements in Sabinene and $\alpha$-Thujene
}

\author{
TORB J ÖRN NORIN and LEIF - A KE SMEDMAN \\ Department of Organic Chemistry, Royal Institute of Technology, S-100 44 \\ Stockholm 70, Sweden
}

\begin{abstract}
The acid-catalysed hydration of $(+)$-sabinene $\left(1 ;[\alpha]_{D}+103^{\circ}, c\right.$ 1.0 in chloroform) using perchloric acid in aqueous dioxane yields (+)-terpinenol-4 $\left(3 ;[\alpha]_{\mathrm{D}}+32.0, c 20.1\right.$ in chloroform), cis- and transsabinene hydrate ( 8 and 9 , respectively), terpinolene (7), $\alpha$ - and $\gamma$ terpinene (5 and 6 , respectively), together with trace quantities of $p$-cymene and unidentified materials.

There is a high degree of stereospecificity $(80 \%)$ in the formation of terpinenol-4 (3) from (+)-sabinene. Thus a classical charge-localized ion can be ruled out as a main product-determining intermediate. The proportions of the two sabinene hydrates ( 8 and 9 ) formed in low yields indicate that the reaction to a large extent is governed by delocalisation effects rather than by steric control.

The hydration of (-)- $\alpha$-thujene (2) proceeds at a much lower rate than that of sabinene, but yields the same products as sabinene, and in the same relative proportions. The products are thus derived from cyclopropylcarbinyl intermediates, and not from a trishomocyclopropenyl cation or a classical relative.
\end{abstract}

\begin{abstract}
A $\mathrm{n}$ important feature in the solvolysis of cyclopropylcarbinyl derivatives is the unique steric course of the reaction. Among other evidence, the stereochemistry of the rearrangement shows that there must be extensive charge delocalisation from the carbinyl carbon to the cyclopropane ring. ${ }^{1-3}$ However, the nature and the extent of this delocalisation is still not clear.

In this communication, we wish to report some results on the rearrangement during acid-catalysed hydration of $(+)$-sabinene $(1)$ and $(-)$ - $\alpha$-thujene (2). The hydrations of these two compounds are simple model reactions for stereochemical studies, since the absolute configurations of the two compounds ${ }^{4-6}$ and of their rearrangement product terpinenol-4 (3) ${ }^{7}$ are known. Thus the stereospecificity in the reaction can be determined. The present investigation was undertaken to compare the results with those of an extensive investigation on the acid-catalysed addition of methanol and acetic acid to bicyclo[3.1.0]hexene-2 (4), ${ }^{8}$ in order to gain additional information concerning the stereospecificity of the rearrangement.
\end{abstract}


The formation of terpinenol-4 (3) upon acid-catalysed hydration of sabinene (1) was discovered by Wallach ${ }^{9}, 10$ in the beginning of this century. More recently, this reaction has been reinvestigated, using gas liquid chromatography (GLC), in order to select the optimum conditions for the formation of terpinenol-4.11 In this latter investigation, the reaction mixture was distilled, and an optically active fraction $\left([\alpha]_{D}+15.2^{\circ}\right.$, homogeneous) of terpinenol-4 was obtained. The homogeneity of this fraction was not determined. However, the result indicates some stereospecificity in the reaction.

In the present investigation, the hydrations were performed in aqueous dioxane $(75 \%)$, using perchloric acid as catalyst $(0.02 \mathrm{~N})$. The reaction mixtures were analysed by means of GLC. Some of the components were identified, using a combined GLC-mass spectrometer. The results of the product study are summarised in Table 1 .

Table 1. Acid-catalysed hydrations of sabinene and $\alpha$-thujene.

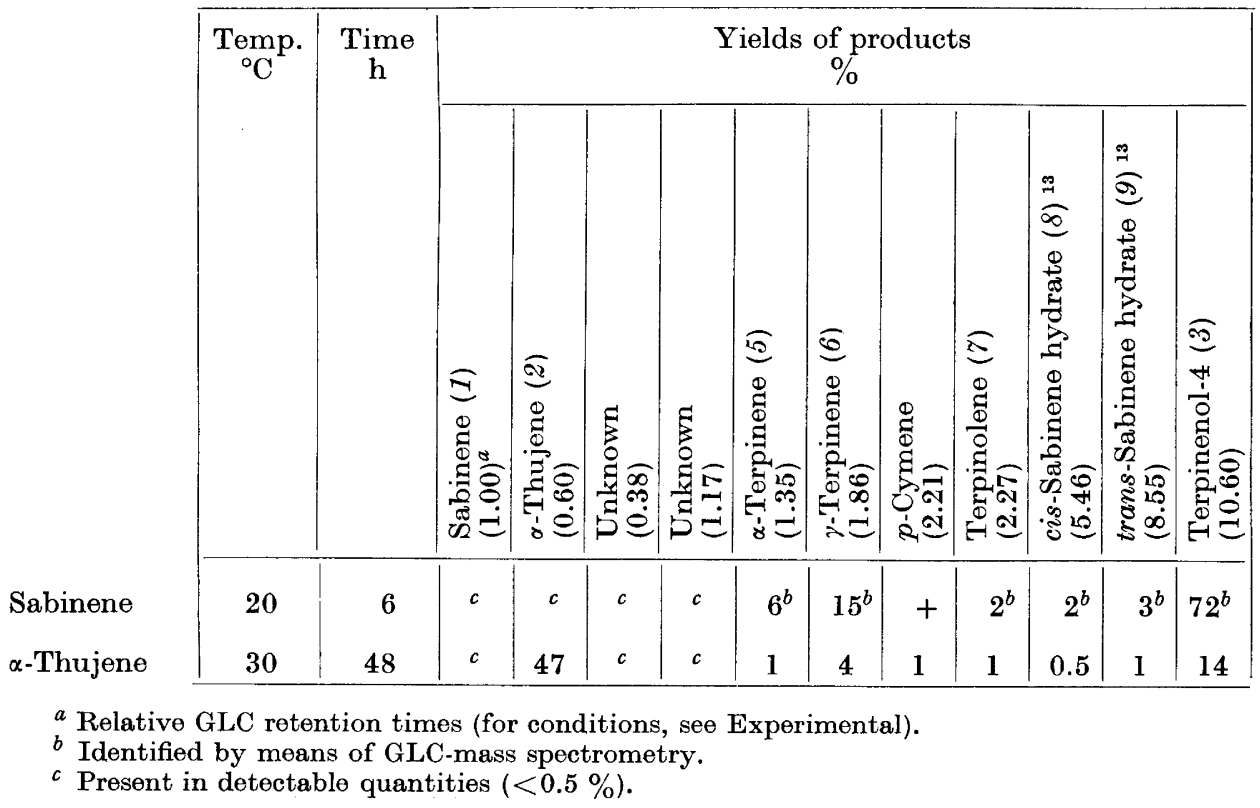

In a preparative experiment, using the conditions described above, a homogeneous terpinenol-4 fraction $\left([\alpha]_{\mathrm{D}}+32.0^{\circ}, c 20.0\right.$ in chloroform) was isolated by chromatography on deactivated alumina, followed by a final purification by preparative GLC. The alcohol was found to be stable upon prolonged treatment under the above reaction conditions, and no change in its optical rotation could be observed. The contribution from a secondary reaction, in which racemic terpinenol-4 is formed from terpinenes, must be very small, since only traces of the alcohol $(<0.5 \%)$ could be detected in 
the reaction product, when a terpinene mixture was treated under the same conditions as those of the above preparative experiment.

From chemical transformations of $(+)$-sabinene $(1)$ to $(+)-\alpha$-methyl- $\alpha-$ isopropylglutaric and $(+)-\alpha$-methyl- $\alpha$-isopropylsuccinic acids, and to other terpenoids, ${ }^{7-9}$ it follows that the $(+)$-sabinene $\left(1 ;[\alpha]_{\mathrm{D}}+103^{\circ}, c 1.0\right.$ in chloroform), used in this investigation, must be of high optical purity $(<2 \%$ of the levorotatory form).

$(+)$-Terpinenol-4 (3) of different optical purities have been reported in the literature. The highest rotation $\left([\alpha]_{\mathrm{D}}+41.9^{\circ}, c 20.0\right.$ in chloroform; $[\alpha]_{D}+48.3^{\circ}$, homogeneous) is recorded for a sample, obtained from oil of lavender. ${ }^{12}$ A value of similar magnitude $\left([\alpha]_{D}+46.67^{\circ}\right.$, homogeneous) for an optically pure sample of the alcohol has been calculated by comparison with compounds in the menthane series of known optical purity, to which terpinenol4 has been chemically transformed. ${ }^{10}$ The above results show that the hydration of sabinene to form terpinenol-4 proceeds with a high degree of stereospecificity $(>80 \%)$. Evidently, some racemization $(15-20 \%)$ also occurs.

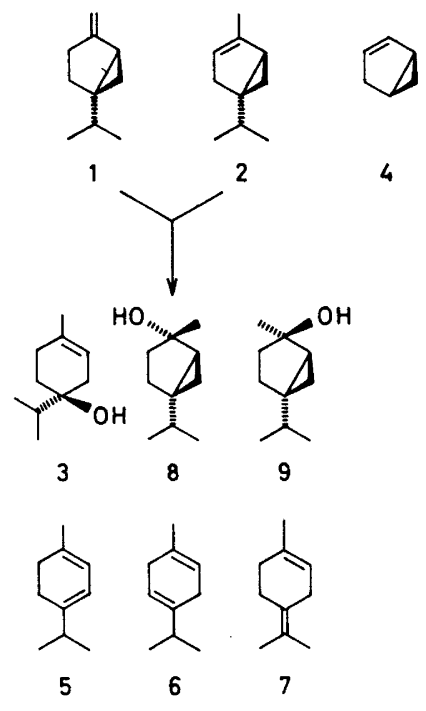

The acid-catalysed additions of methanol and of acetic acid to bicyclo[3.1.0]hexene-2 (4) have been discussed in detail by Freeman et al. ${ }^{8}$ The acidcatalysed hydration of $(+)$-sabinene (1) and (-)- $\alpha$-thujene (2) proceeds in a way analogous to the solvolysis of the above unsubstituted hydrocarbon (4). It is interesting to note that the hydration of sabinene proceeds much faster than that of $\alpha$-thujene.

A 3-carbonium ion, such as a trishomocyclopropenyl cation (10) or a classical relative (11), could be ruled out as a product determining intermediate in the electrophilic addition of methanol or acetic acid to bicyclo[3.1.0]hexene-2 (4). ${ }^{11}$ The hydration of $\alpha$-thujene similarly yields products which are derived from cyclopropylcarbinyl intermediates, and not from a homocyclopropyl- 
carbinyl cation. The products are the same and in the same proportions as those from sabinene.

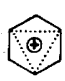

10<smiles>C1CC2CCC1C2</smiles>

11<smiles>CO[C@@H]1CCC2CCC21</smiles>

12<smiles>CO[C@H]1CC2CCC1C2</smiles>

13

From the product composition of the kinetically controlled addition of methanol to bicyclo[3.1.0]hexene-2 ${ }^{11}$ it is evident that the steric course of the reaction must be influenced by delocalisation effects in transition or intermediate states, since the initial proportion of cis- and trans-2-methoxybicyclo[3.1.0]hexanes (12) and (13) is calculated to be 45:55. A sterically controlled addition to a classical charge localised ion should yield more of the trans-isomer (13). The proportions of the two sabinene hydrates, formed upon hydration of sabinene, similarly indicate that the reaction is governed mainly by delocalisation effects, rather than by steric control (for sterically controlled reactions, see Ref. 13), via the classical charge-localised ion (14).

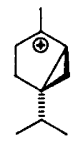

14

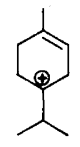

15

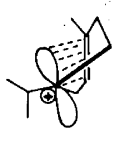

16

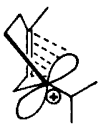

17

The stereospecificity in the formation of (+)-terpinenol-4 (3) from (+)sabinene $(1)$ is in complete agreement with previous examples of cyclopropylcarbinyl rearrangements in more rigid systems. ${ }^{1-5}$ Thus there must be extensive charge delocalisation in the rearrangement and a classical charge-localised ion (15) can be ruled out as a main product-determining intermediate. However, some racemic alcohol is also formed, which thus would suggest that there is a contribution from a classical charge-localised ion (15), or that two sets of interconvertible charge-delocalised ions (16) and (17) are involved in the reaction.

\section{EXPERIMENTAL}

GLC analyses were performed on an Aerograph 204B instrument, using a Carbowax $20 \mathrm{M}$ column $\left(10 \%\right.$ on Chromosorb W $60-80$ mesh; $\left.10^{\prime} \times 1 / 8^{\prime \prime} ; 120^{\circ}\right)$. Relative retention times for the hydration products are given in Table 1 (sabinene, 1.00). The compositions of the product mixtures are calculated from peak areas. Corrections for differences in detector response were made for sabinene and terpinenol-4. The mass spectrometric identifications were made on an LKB 9000 combined GLC-mass spectrometer, using a Carbowax $20 \mathrm{M}$ column.

Acid-catalysed hydrations. (+)-Sabinene $\left(0.5 \mathrm{~g} ;[\alpha]_{\mathrm{D}}+103^{\circ}, c 1.0\right.$ in chloroform) was treated with perchloric acid $(0.02 \mathrm{~N})$ in dioxane/water $(3: 1 ; 50 \mathrm{ml})$ for $6 \mathrm{~h}$. The reaction mixture was poured into ice/water, and extracted with light petroleum (b.p. $35-45^{\circ}$ ). The organic material was analysed by GLC and by GLC-mass spectrometry (Table 1), and the various constituents were identified by comparisons with authentic compounds or mixture of compounds.

The hydration product was chromatographed on alumina (standard, activity II - III). Ether $(5 \%)$ in light petroleum (b.p. $35-45^{\circ}$ ) eluted a terpinenol-4 fraction which ac-

Acta Chem. Scand. 25 (1971) No. 6 
cording to GLC contained small amounts of sabinene hydrates. This fraction was further purified by preparative GLC (Aerograph Autoprep A 700; $10 \%$ QF-1 on 60-80 mesh Chromosorb $\mathrm{W}$; column dimensions $20^{\prime} \times 3 / 8^{\prime \prime}$; column temp. $45^{\circ}$ ) to yield homogeneous terpinenol-4, $[\alpha]_{\mathrm{D}}+32.0^{\circ}$ (c 20.0 in chloroform).

The hydration of $\alpha$-thujene was performed, using the same conditions as described above for sabinene (reaction time, $48 \mathrm{~h}$; temp. $30^{\circ}$ ); the product analysis is shown in Table 1 .

\section{REFERENCES}

1. Winstein, S. and Kosower, E. M. J. Am. Chem. Soc. 81 (1959) 4399.

2. Mazur, R. H., White, W. N., Semenov, D. A., Lee, C. C., Silver, M. S. and Roberts, J. D. J. Am. Chem. Soc. 81 (1959) 4390.

3. Birladeanu, L., Hanafuse, T., Jonson, B. and Winstein, S. J. Am. Chem. Soc. 88 (1966) 2316.

4. Norin, T. Acta Chem. Scand. 16 (1962) 640.

5. Ohloff, G., Uhde, G., Thomas, A. F. and Kovats, E. sz. Tetrahedron 22 (1966) 309.

6. Cox, M. R., Koch, H. P., Whalley, W. B., Hursthouse, M. B. and Rogers, D. Chem. Commun. 1967212.

7. Ohloff, G. and Uhde, G. Helv. Chim. Acta 48 (1965) 10.

8. Freeman, P. K., Grostic, M. F. and Raymond, F. A. J. Org. Chem. 30 (1965) 771.

9. Wallach, O. Ann. 350 (1906) 165.

10. Wallach, O. Ann. 360 (1908) 98.

11. Tolstikov, G. A., Lishtvanova, L. N. and Goryaev, M. I. Zh. Obshch. Khim. 33 (1963) 683; cf. J. Gen. Chem. (USSR) 33 (1963) 676.

12. Naves, R.-R. and Tullan, P. Bull. Soc. Chim. France 19602123.

13. Daly, J. W., Green, F. C. and Eastman, R. H. J. Am. Chem. Soc. 80 (1958) 6330.

Received October 26, 1970. 